\title{
Scalp lesions and hair loss
}

\author{
David A. Whiting, MD
}

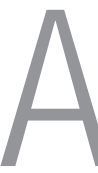

17-year-old white male complained of scalp problems for the preceding 8 months. Initially he developed a painful lesion, which looked like a blood blister, on the right posterior crown. It was believed to be a wrestling injury and was treated with topical antiseptics. This patient was an expert wrestler and competed frequently. At some point his tinea pedis (athlete's foot) was treated with terbinafine. However, the scalp lesion did not improve.

After 5 months he was sent to a dermatologist. At that stage he had no obvious hair loss and was thought to have eczema. He was treated with topical clobetasol lotion, which did not help. He was also given bupropion and duloxetine for depression. Two months later he developed obvious, painful hair loss, which spread over the crown. A scalp biopsy showed folliculitis decalvans, and he was treated with oral clindamycin and rifampicin. Despite this therapy, the pain, abscess formation, and hair loss continued.

A month later, he was seen at the Hair and Skin Research and Treatment Center at Baylor University Medical Center for further evaluation. He was tired, ill, depressed, and in pain. His scalp condition was continuing to deteriorate. The patient appeared listless and feverish. He had extensive and patchy hair

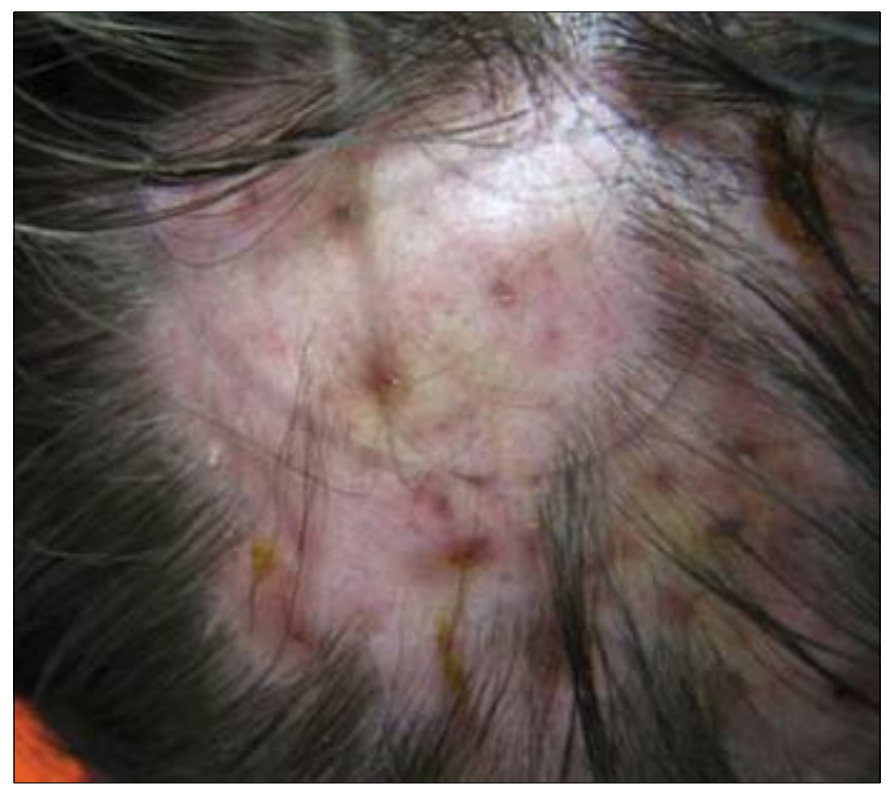

Figure 1. Hair loss and inflammation. loss over the crown measuring $7 \times 12$ $\mathrm{cm}$ (Figure 1). Abscesses and scarring were present. Some lesions were purulent, and some cervical lymphadenopathy was found. The results of Wood's light examination, potassium hydroxide examination of hair shafts, bacterial culture, blood work, and a comprehensive metabolic panel were normal. A fungal culture showed scanty growth of Trichophyton tonsurans.

A 4-mm punch biopsy of the scalp sectioned horizontally showed considerable reduction in terminal hairs and a relative increase in vellus-like hairs,
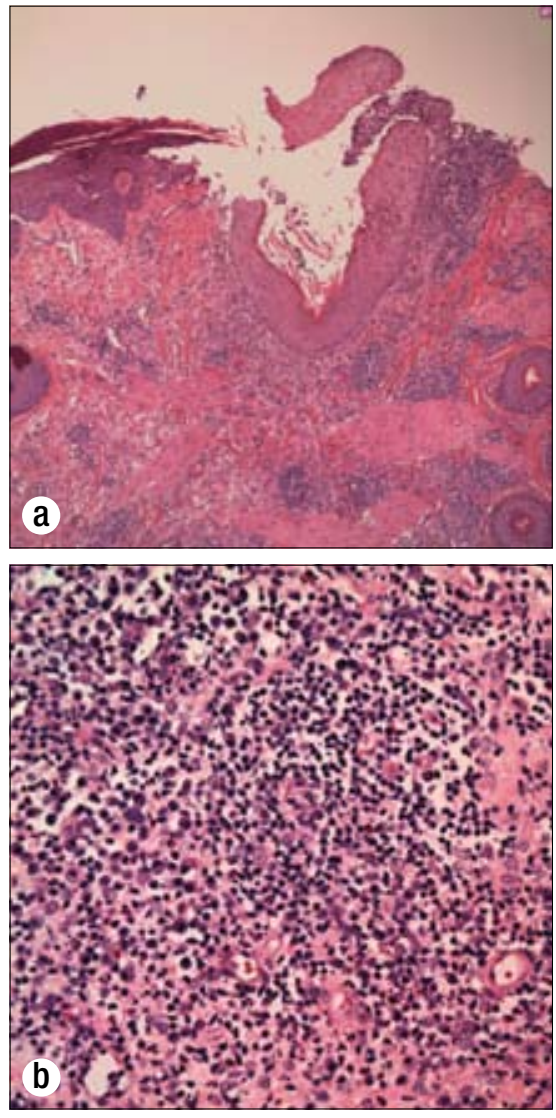

Figure 2. Scalp biopsy showing (a) hyperkeratosis, parakeratosis, infundibular disruption, and perifollicular, lymphohistiocytic infiltrates and (b) granulomatous infiltration of neutrophils, eosinophils, plasma cells, and foreign body giant cells. Hematoxylin and eosin stain, $\times 100$.

with $75 \%$ of the terminal hairs in telogen phase. A dense, dermal infiltrate was seen with abscess formation in the upper dermis, comprising lymphocytes, histiocytes, neutrophils, eosinophils, plasma cells, and foreign body giant cells (Figure 2). No obvious

From the Hair and Skin Research and Treatment Center, Baylor University Medical Center, Dallas, Texas.

Corresponding author: David A. Whiting, MD, Hair and Skin Research and Treatment Center, 3600 Gaston Avenue, Wadley Tower, Suite 1058, Dallas, Texas 75246 (e-mail: whiting@hairskinrtc.com). 
fungal spores or hyphae were seen. Five terminal infundibula were empty. There was moderate fibrosis around some upper follicles. Periodic acid-Schiff and Gomori's methenamine silver stains were negative for fungi, but the overall appearance was compatible with a fungal infection.

DIAGNOSIS: Tinea capitis with kerion formation due to Trichophyton tonsurans.

Fine-particle griseofulvin, given at $250 \mathrm{mg}$ twice a day for 6 months, produced an apparent cure. Scattered scarring remained, but satisfactory hair coverage had been obtained (Figure 3). The patient felt cheerful and well and had resumed competitive wrestling.

Kerion, a pruritic mass of inflamed tissue that develops in response to fungal infections in the hair follicles, needs to be considered in cases of resistant pyodermas of the scalp. It can be difficult to diagnose. Our patient, a wrestler, was in close contact with other wrestlers, and fungal, bacterial, viral, and parasitic infections can be easily transmitted in that context.

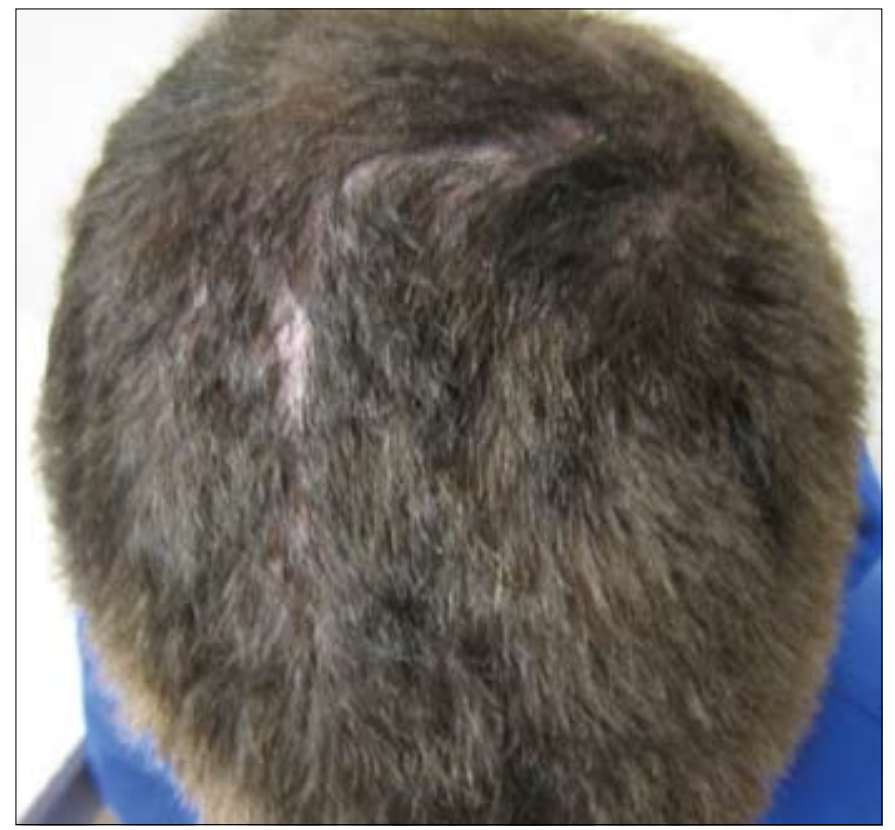

Figure 3. Hair regrowth following treatment with griseofulvin. 